\title{
Transcriptome analysis of leaves, roots and flowers of Panax notoginseng identifies genes involved in ginsenoside and alkaloid biosynthesis
}

\author{
Ming-Hua Liu ${ }^{1 \dagger}$, Bin-Rui Yang ${ }^{3 \dagger}$, Wai-Fung Cheung ${ }^{4,6 \dagger}$, Kevin Yi Yang ${ }^{1,2}$, He-Feng Zhou ${ }^{3}$, Jamie Sui-Lam Kwok , \\ Guo-Cheng Liư ${ }^{4}$ Xiao-Feng Li ${ }^{4}$, Silin Zhong ${ }^{5}$, Simon Ming-Yuen Lee ${ }^{3^{*}}$ and Stephen Kwok-Wing Tsui ${ }^{1,2^{*}}$
}

\begin{abstract}
Background: Panax notoginseng (Burk.) F.H. Chen is one of the most highly valued medicinal plants in the world. The major bioactive molecules are triterpene saponins, which are also known as ginsenosides. However, its large genome size has hindered the assembly of a draft genome by whole genome sequencing. Hence, genomic and transcriptomic details about $P$. notoginseng, especially its biosynthetic pathways and gene expression in different parts of the plant, have remained largely unknown until now.

Results: In this study, RNA sequencing of three different $P$. notoginseng tissues was performed using next generation DNA sequencing. After assembling the high quality sequencing reads into 107,340 unigenes, biochemical pathways were predicted and 9,908 unigenes were assigned to 135 KEGG pathways. Among them, 270 unigenes were identified to be involved in triterpene saponin biosynthesis. In addition, 350 and 342 unigenes were predicted to encode cytochrome P450s and glycosyltransferases, respectively, based on the annotation results, some of which encode enzymes responsible for the conversion of the triterpene saponin backbone into different ginsenosides. In particular, one unigene predominately expressed in the root was annotated as CYP716A53v2, which probably participates in the formation of protopanaxatriol from protopanaxadiol in $P$. notoginseng. The differential expression of this gene was further confirmed by real-time PCR.

Conclusions: We have established a global transcriptome dataset for $P$. notoginseng and provided additional genetic information for further genome-wide research and analyses. Candidate genes involved in ginsenoside biosynthesis, including putative cytochrome P450s and glycosyltransferases were obtained. The transcriptomes in different plant tissues also provide invaluable resources for future study of the differences in physiological processes and secondary metabolites in different parts of $P$. notoginseng.
\end{abstract}

Keywords: Panax notoginseng, Transcriptome, Ginsenosides

\section{Background}

Panax notoginseng (Burk.) F.H. Chen, which is popularly known as Sanqi or Tienchi Ginseng, is a species of the Panax genus in the Araliaceae family [1]. P. notoginseng has been cultivated for about 400 years in China. It was previously considered a variety of Panax pseudo-ginseng,

\footnotetext{
*Correspondence: simonlee@umac.mo; kwtsui@cuhk.edu.hk

${ }^{\dagger}$ Equal contributors

${ }^{3}$ State Key Laboratory of Quality Research in Chinese Medicine and Institute of Chinese Medical Sciences, Macao, China

'School of Biomedical Sciences, The Chinese University of Hong Kong, Hong Kong, China

Full list of author information is available at the end of the article
}

but in 1948 it was defined as an independent species of the Panax genus by Chen Feng-Huai and is now officially named Panax notoginseng (Burk.) F.H. Chen. Nowadays, more than $85 \%$ of the $P$. notoginseng in the worldwide market is produced in the city of Wenshan, Yunnan Province, China.

P. notoginseng is present in several famous traditional Chinese medicinal products, such as Yunnan Bai Yao (a remedy for injury induced by trauma and bleeding) and Pian Zai Huang (a remedy for relieving pain and detoxification). It is also famous for its haemostatic properties [2]. The classification of $P$. notoginseng from the American

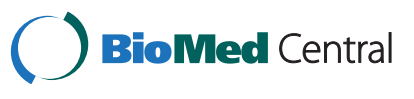

(C) 2015 Liu et al. This is an Open Access article distributed under the terms of the Creative Commons Attribution License (http://creativecommons.org/licenses/by/4.0), which permits unrestricted use, distribution, and reproduction in any medium, provided the original work is properly credited. The Creative Commons Public Domain Dedication waiver (http:// creativecommons.org/publicdomain/zero/1.0/) applies to the data made available in this article, unless otherwise stated. 
Herbal Products Association is Class 2b and it is indicated in pregnancy because of possible haemostatic effects. It was reported that $P$. notoginseng extract administered to rats after cerebral ischaemia reduced infarct volume and inhibited inflammatory inhibitors such as inducible nitric oxide synthase and cyclooxygenase 2 via blocking of the NF- $\kappa B$ pathway [3], suggesting a neuroprotective effect. Moreover, saponins of $P$. notoginseng extract were able to modulate the expression of caspases and attenuate apoptosis in rats following focal cerebral ischaemia-reperfusion [4]. In KK-Ay diabetic mice injected with $P$. notoginseng extract, significantly lowered fasting blood glucose levels, improved glucose tolerance and lighter body weights were observed [5]. Besides the roots of $P$. notoginseng, total saponins extracted from caudexes and leaves have been commonly used for improving mental function, treating insomnia, and alleviating anxiety [6]. The flower buds of $P$. notoginseng are also used in clinics for treating hypertension, vertigo, tinnitus and acute faucitis in China [7].

Chemically, the main bioactive compounds found in $P$. notoginseng are saponins, which have diverse biological activities such as membrane-permeabilising, immunostimulating, hypocholesterolemic, anti-carcinogenic, and anti-microbial activities [8-10]. The P. notoginseng-derived triterpene saponins include 20(S)-protopanaxadiol and 20 (S)-protopanaxatriol, which exhibit opposing wound healing and anti-tumour actions on the vascular system [11]. Notably, $P$. notoginseng also shares many similar chemical constituents with Asian ginseng (P. ginseng C.A. Mey) and American ginseng (P. quinquefolius L.) [12]. These Panax species have species-specific saponin constituents, e.g. pseudo-ginsenoside F11 is unique to American ginseng whereas ginsenoside Rg3 is only present in Asian ginseng [13]. More than 60 chemotypes of $P$. notoginseng classified according to the accumulation of different ginsenosides in roots, leaves and flowers have been reported $[11,14]$. $P$. notoginseng contains significantly higher amounts of ginsenosides Rg1 and Rb1 compared with other ginseng species, and the ratios of $\operatorname{Rg} 1 / \operatorname{Re}$ and $\operatorname{Rg} 1 / \operatorname{Rb} 1$ in $P$. notoginseng are the highest among ginseng species. In particular, notoginsenoside $\mathrm{R} 1$ has been identified in $P$. notoginseng but is absent in other ginseng species.

Several previous studies have suggested that the precursor molecules for triterpene saponin biosynthesis are isoprenoids, which are synthesized via the mevalonic acid (MVA) pathway, leading to the biosynthesis of 2,3oxidosqualene [15]. This central molecule is then modified through various biochemical reactions of its triterpene skeleton, resulting in the production of various ginsenosides. Notably, ginsenosides can be isolated from different parts of $P$. notoginseng, e.g. the underground parts of $P$. notoginseng are rich in protopanaxatriol- and protopanaxadiol-type saponins, while leaves and flowers contain protopanaxadiol-type saponins only. On the other hand, the ginsenosides $\mathrm{Rc}, \mathrm{Rb} 2$ and $\mathrm{Rb} 3$ are relatively abundant in aerial parts, compared with the underground parts of $P$. notoginseng $[11,16]$. Although considerable research has been done on the pharmacological activities of ginsenosides, to date very little is known about the ginsenoside biosynthetic pathway. Some candidate genes likely to be involved in hydroxylation or glycosylation of aglycones for triterpene saponin biosynthesis are cytochrome P450s (CYP450) and glycosyltransferases (GT), but no candidate has been identified for the cyclization step [17].

In addition, $P$. notoginseng is a shade plant and is commonly cultivated in mountain areas of Wenshan at altitudes of $1200-2000 \mathrm{~m}$ around $23.5^{\circ} \mathrm{N}, 104^{\circ} \mathrm{E}$ [18]. Because of the humid and warm environment, $P$. notoginseng is easily infected by pests and diseases, especially in the roots. Alkaloids, which have been identified in more than 4,000 plant species, play a role in protecting plants from pathogen and pest damage [19]. However, alkaloid-related genes in $P$. notoginseng have not been reported previously.

Transcriptomic and genomic data for $P$. notoginseng are very limited despite the pharmacological importance of this plant. Considering there are thousands of genes in its genome, only 435 mRNA sequences originating from $P$. notoginseng could be retrieved from the nucleotide databases of the National Centre for Biotechnology Information (NCBI). Over the last decade, next-generation DNA sequencing technology has provided a rapid and economical way to study the gene expression profiles of plant species. In this study, we established transcript databases for leaves, roots and flowers from 3-year-old P. notoginseng. Moreover, we identified genes encoding enzymes involved in triterpene saponin and alkaloid biosynthesis. Differentially expressed CYP450s and GTs in the three tissues are also reported.

\section{Results and discussion}

\section{Sequencing and de novo assembly}

To study the transcriptomes of $P$. notoginseng, leaves, roots and flowers were collected from 3-year-old plants. Total RNA was extracted from each part and then mRNA was isolated. Each sample was sequenced using the Illumina $\mathrm{HiSeq}^{\mathrm{rm}} 2000$ platform. Sequencing yielded approximately 213 million 90-base pair (bp) paired end raw reads, or approximately $19 \mathrm{Gbp}$ in total. We filtered out adapter sequences and reads that were shorter than $50 \mathrm{bp}$, and ultimately generated 5.8, 5.8 and 6.1 Gbp of high-quality (HQ) reads for leaves, roots and flowers, respectively. All of the HQ sequencing reads from the three organs were deposited in NCBI and can be accessed in the Sequence Read Archive (SRA) under the BioProject accession number PRJNA228978. The HQ reads of each sample library were assembled using the Trinity software [20] and the TGI Clustering Tool 
(TGICL) [21], followed by the Phrap assembler [22] to remove redundant Trinity-generated contigs. Finally, $128,665,94,258$ and 124,888 unigenes were obtained for leaves, roots and flowers, respectively. We also pooled the reads from all three organs together and repeated the above steps, resulting in 205,000 contigs and 107,340 unigenes with a mean length of $781 \mathrm{bp}$ and $1,039 \mathrm{bp}$, respectively. The length distribution of contigs and unigenes is shown in Additional file 1. A summary of sequencing and assembly results is shown in Table 1 .

Compared with a separate study of 4-year-old $P$. notoginseng [17], which generated 30,852 unigenes $(14,005$ contigs with mean length $581 \mathrm{bp}$ and 16,847 singletons with mean length $343 \mathrm{bp}$ ), we present three times more and much longer unigenes from $P$. notoginseng roots $(107,340$ unigenes with mean length $1,039 \mathrm{bp})$. This is probably because of a 48 -fold increase in sequencing throughput in this study. The large number of novel unigenes should cover the majority of genes in the $P$. notoginseng genome and provide a useful resource for future studies on this pharmacologically important plant.

\section{Annotation and differential expression of transcripts in different tissues}

All unigenes from leaves, roots and flowers were annotated separately using BLAST searches against the NCBI nonredundant protein $(\mathrm{Nr})$, UniProt protein, The Arabidopsis Information Resource (TAIR), tomato genome (ITAG) and PlantCyc public databases. Detailed counts of the annotated unigenes are presented in Additional file 2. In total, there were 71,212, 58,182 and 75,404 annotated unigenes for leaves, roots and flowers, respectively, with at least one significant match in the aforementioned public databases. Figure 1 shows the number of unigenes annotated by all databases. The annotation percentage for $P$. notoginseng unigenes $(74,839$ out of $107,340,69.72 \%)$ was much higher than that for $P$. ginseng unigenes $(94,535$ out of 178,145 , $53.06 \%$ ) [23]. When the unigenes from different $P$. notoginseng tissues were compared, we found that 41,373 unigenes were shared by all three tissues (Figure 2). On the other hand, 8,858, 5,814 and 10,649 unigenes were specifically found in leaves, roots and flowers, respectively, with flowers having the highest number of unique unigenes.

We next investigated the transcriptomic similarities and differences between $P$. notoginseng and $P$. ginseng using BLAST searches of $P$. notoginseng unigenes against $P$. ginseng unigenes, with an E-value threshold of 1e-10 [23]. P. ginseng transcriptome contigs were obtained under accession number GAAG00000000 from the NCBI. The results showed that 77,470 unigenes of $P$. notoginseng had at least one match to the contigs of $P$. ginseng, suggesting that many of our unigenes are $P$. notoginseng-specific. Next, proteins encoded by our unigenes were predicted by the Trinity software and orthologous groups were identified using OrthoMCL [24], which groups orthologous proteins based on sequence similarity. A total of 64,742 protein sequences from 107,340 unigenes were clustered into 9,949 orthologous groups for $P$. notoginseng. For $P$. ginseng, a total of 29,289 protein sequences from 67,786 contigs were clustered into 8,959 orthologous groups. We found that 8,424 groups were common to both species, with 1,525 orthologous groups specific to P. notoginseng, and 535 groups specific to $P$. ginseng.

To evaluate the abundance of transcripts in specific organs, the high quality reads were mapped back to the transcriptome generated from all three plant tissues. Among the top 10 most expressed unigenes in the root transcriptome (Additional file 3), one of the most abundant genes was dammarenediol-II synthase (DS), which is an important player in triterpene saponin biosynthesis

Table 1 Summary of Illumina sequencing and assembly of $P$. notoginseng

\begin{tabular}{|c|c|c|c|c|}
\hline & Leaves & Roots & Flowers & Three tissues together \\
\hline Number of $\mathrm{HQ}$ reads & $65,041,040$ & $65,258,974$ & $68,125,310$ & $198,425,324$ \\
\hline Length of $\mathrm{HQ}$ reads (bp) & $5,853,693,600$ & $5,873,307,660$ & $6,131,277,900$ & $17,858,279,160$ \\
\hline Number of contigs & 167,954 & 140,265 & 184,389 & 205,000 \\
\hline Length of contigs (bp) & $56,302,711$ & $48,125,902$ & $60,389,472$ & $160,205,000$ \\
\hline Average length of contigs (bp) & 335 & 343 & 328 & 781 \\
\hline N50 of contigs (bp) & 537 & 606 & 539 & 1,218 \\
\hline Number of unigene* & 128,665 & 94,258 & 124,888 & 107,340 \\
\hline Length of unigene (bp) & $76,176,975$ & $60,684,037$ & $92,995,924$ & $111,620,756$ \\
\hline Average length of unigene (bp) & 592 & 644 & 745 & 1,039 \\
\hline N50 of unigene (bp) & 912 & 1,025 & 1,232 & 1,526 \\
\hline Number of clusters & 50,984 & 37,727 & 54,977 & 76,938 \\
\hline Number of singletons & 77,681 & 56,531 & 69,911 & 30,402 \\
\hline
\end{tabular}

*Total number of clusters and singletons. 


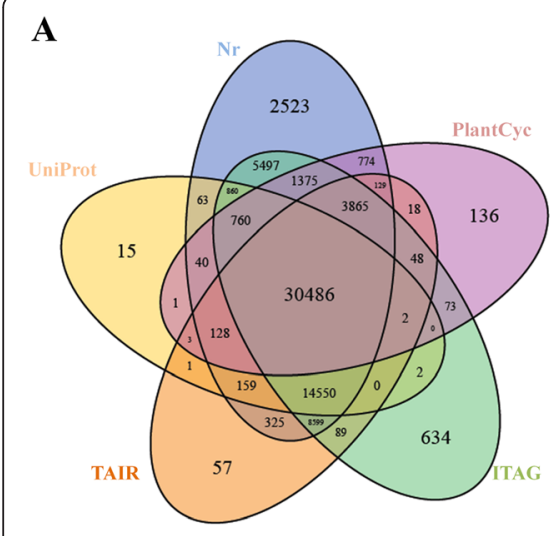

Leaves
B

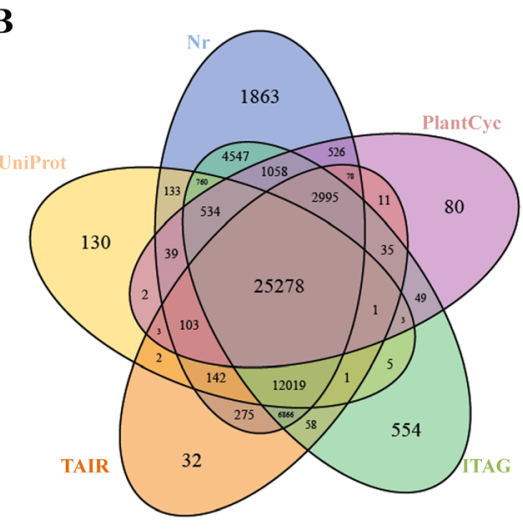

Roots
C

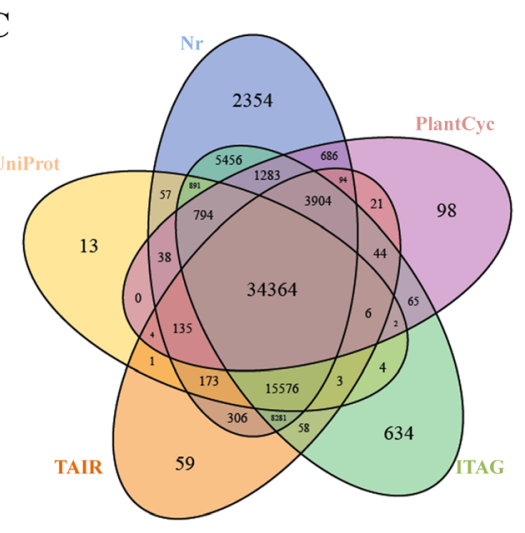

Flowers

Figure 1 Venn diagram of unigenes from leaves, roots and flowers annotated using various databases. The Venn diagram shows the overlapping unigenes annotated in the Nr, Uniprot, TAIR, ITAG and PlantCyc databases in leaves (A), roots (B) and flowers (C).

in $P$. notoginseng [25]. In addition, unigenes encoding cytochrome P450 CYP716A47, which is involved in the conversion of protopanaxadiol from dammarenediol-II, were also present at a very high level. This is consistent with a previous report about the co-expression of DS and CYP716A47 in P. ginseng [26]. Last but not least, reticuline oxidase-like protein-like isoform 1 is a predicted protein with sequence similarity to reticuline oxidase, which is involved in forming benzophenanthridine alkaloids as a pathogenic attack response.

\section{Identification of genes involved in triterpene saponin} biosynthesis

The Kyoto Encyclopaedia of Genes and Genomes (KEGG) is a tool for functional classification and pathway assignment based on gene-associated biochemical pathways. In

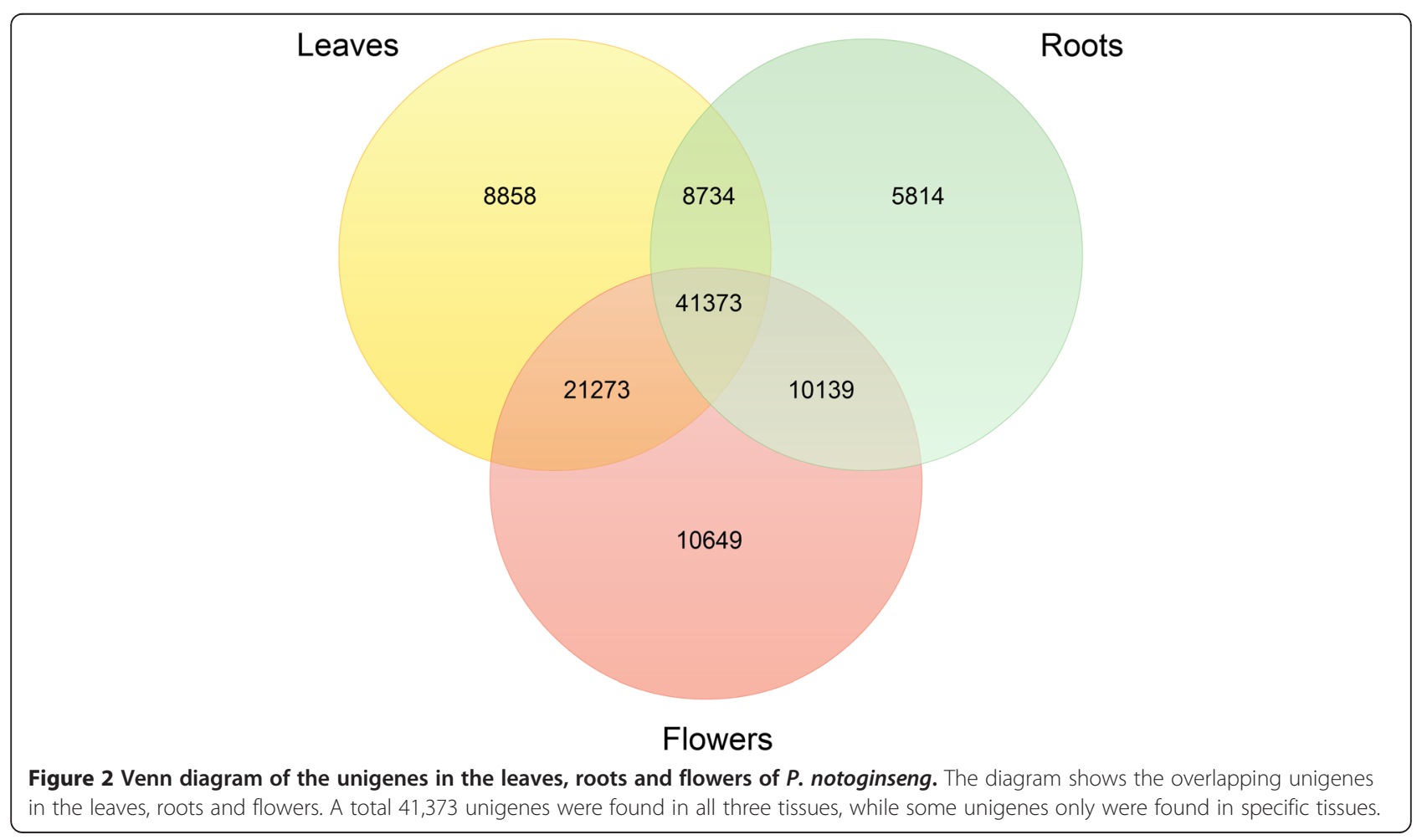


total, 9,908 unigenes from all three tissues having enzyme commission numbers were assigned to 135 KEGG pathways (Additional file 4). The cluster for metabolism represented the largest group, with most unigenes involved in carbohydrate metabolism, amino acid metabolism, nucleotide metabolism and metabolism of cofactors and vitamins. Among them, 616 unigenes were involved in the biosynthesis of various secondary metabolites (Table 2), with unigenes involved in phenylpropanoid biosynthesis forming the largest group, followed by terpenoid backbone biosynthesis.

Triterpene saponins are synthesized by terpenoid backbone biosynthesis, followed by sesquiterpenoid and triterpenoid biosynthesis. According to the putative pathway, specific CYP450s and GTs are involved in the formation of various ginsenosides (Figure 3). From our annotation results, 270 unigenes were identified to encode all of the known enzymes involved in triterpene saponin biosynthesis. There were multiple unigenes annotated to the same enzyme, which may represent different members of the same gene family. Table 3 shows the reads per kilobase of transcript per million reads mapped (RPKM) values of genes encoding enzymes involved in triterpene saponin biosynthesis in leaves, roots and flowers. The RPKMs of all annotated isoforms for the same gene were summed as the RPKM of that gene. We identified 12 out of 14 fulllength cDNA encoding enzymes involved in triterpene saponin biosynthesis and their corresponding accession numbers in NCBI nucleotide databases are listed in Table 4. A heat map of differentially expressed genes involved in triterpene saponin biosynthesis is shown in Figure 3. Significant differential expression of these genes could be recognized in different $P$. notoginseng tissues, and most of the genes involved in triterpene saponin biosynthesis showed higher expression in roots compared with leaves or flowers. The expression levels of selected genes (8 out of 14) in each part were validated by real-time PCR (Figure 4).

Luo et al. [17] previously analysed the root transcriptome of 4-year-old $P$. notoginseng and discovered many partial cDNAs encoding enzymes involved in triterpene saponin biosynthesis. However, hydroxymethyl glutaryl CoA synthase (HMGS), a key enzyme of triterpene saponin biosynthesis, was absent in their transcriptome. In contrast, the HMGS gene was abundant in our transcriptomes, probably because of the much greater sequencing depth achieved in our study. Therefore, compared with other published transcriptomes, the transcriptomes reported in this study will be more useful for cloning important genes involved in secondary metabolite biosynthetic pathways of $P$. notoginseng. Moreover, $\beta$-amyrin synthase (AS), a key enzyme for oleanane-type ginsenoside biosynthesis, was also found in this study. Notably, AS was discovered in all three tissues of 3-year-old P. notoginseng (Table 3).

Table 2 Number of unigenes related to secondary metabolites in $P$. notoginseng

\begin{tabular}{|c|c|c|c|c|}
\hline Secondary metabolites biosynthesis pathways & Pathway ID & Leaves & Roots & Flowers \\
\hline Anthocyanin biosynthesis & ko00942 & 10 & 7 & 14 \\
\hline Caffeine metabolism & ko00232 & 12 & 12 & 20 \\
\hline Carotenoid biosynthesis & ko00906 & 15 & 5 & 32 \\
\hline Cutin, suberin and wax biosynthesis & ko00073 & 14 & 12 & 15 \\
\hline Diterpenoid biosynthesis & ko00904 & 8 & 3 & 12 \\
\hline Flavone and flavonol biosynthesis & ko00944 & 3 & 2 & 5 \\
\hline Flavonoid biosynthesis & ko00941 & 23 & 21 & 33 \\
\hline Indole alkaloid biosynthesis & ko00901 & 8 & 13 & 11 \\
\hline Isoquinoline alkaloid biosynthesis & ko00950 & 57 & 32 & 51 \\
\hline Limonine and pinene degradation & ko00903 & 13 & 10 & 15 \\
\hline Monoterpenoid biosynthesis & ko00902 & 4 & 6 & 6 \\
\hline Nicotinate and nicotinamide metabolism & ko00760 & 37 & 39 & 48 \\
\hline Phenylpropanoid metabolism & ko00940 & 88 & 100 & 154 \\
\hline Sesquiterpenoid and triterpenoid biosynthesis & ko00909 & 19 & 15 & 13 \\
\hline Steroid biosynthesis & ko00100 & 27 & 18 & 42 \\
\hline Stibenoid diarylhepatanoid and gingerol biosynthesis & ko00945 & 2 & 1 & 2 \\
\hline Terpenoid backbone biosynthesis & ko00900 & 91 & 56 & 56 \\
\hline Tropane, piperidine and pyridine alkaloid biosynthesis & ko00960 & 57 & 47 & 66 \\
\hline Ubiquinone and other terpenoid-quinone biosynthesis & ko00130 & 45 & 20 & 31 \\
\hline Zeatin biosynthesis & ko00908 & 10 & 6 & 14 \\
\hline
\end{tabular}




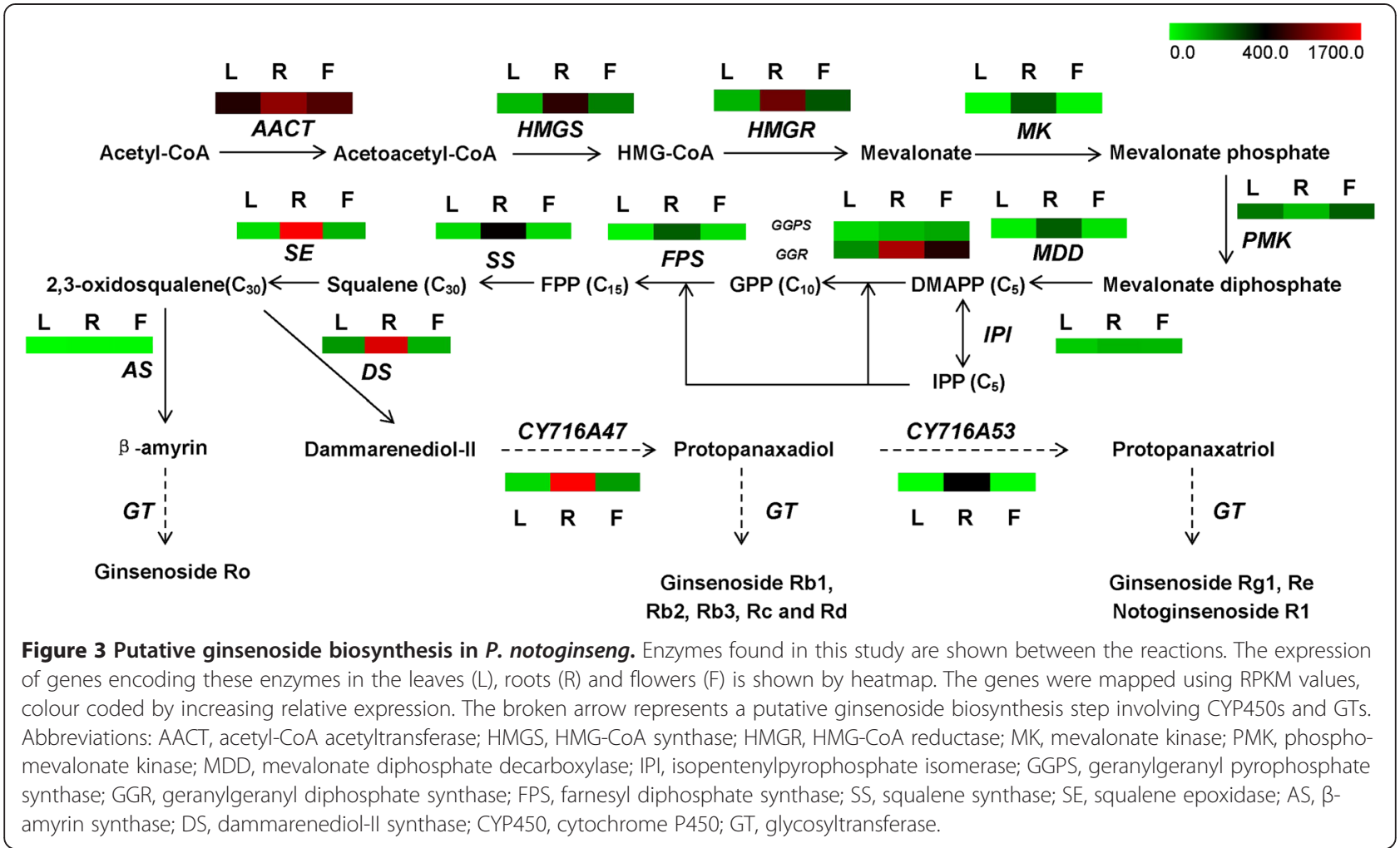

Identification of genes related to biosynthesis of different ginsenosides

There are published reports showing that different parts of $P$. notoginseng differ in the synthesis of triterpene saponins. Roots are rich in protopanaxatriol- and protopanaxadioltype saponins whereas leaves and flowers contain protopanaxadiol-type saponins only [11]. It has been suggested that putative candidate genes involved in triterpene saponins biosynthesis are mainly CYP450s and GTs, which may account for the synthesis and accumulation of triterpene saponins in specific organs $[27,28]$. In this study, 350 and 342 members of the CYP450 and GT gene families, respectively, were identified (Additional files 5 and 6).

It has been reported that the cytochrome $\mathrm{P} 450$ CYP716A53v2 participates in the formation of protopanaxatriol from protopanaxadiol in P. ginseng [29]. In our

Table 3 Discovery of unigenes involved in triterpene saponin biosynthesis in $\boldsymbol{P}$. notoginseng

\begin{tabular}{|c|c|c|c|c|c|}
\hline Enzymes name & EC number & Abbreviation & Leaves & Roots & Flowers \\
\hline Acetyl-CoA acetyltransferase & 2.3.1.9 & $\mathrm{AACT}$ & 587.76 & 1142.49 & 816.36 \\
\hline Hydroxymethyl glutaryl CoA synthase & 2.3.3.10 & HMGS & 110.12 & 634.67 & 200.10 \\
\hline 3-hydroxy-3-methylglutaryl-coenzymeA reductase & 1.1.1.34 & HMGR & 117.75 & 961.07 & 263.98 \\
\hline Mevalonate kinase & 2.7.1.36 & MK & 9.42 & 261.79 & 19.36 \\
\hline Phosphomevalonate kinase & 2.7.4.2 & PMK & 211.09 & 107.73 & 251.65 \\
\hline Mevalonate diphosphosphate decarboxylase & 4.1.1.33 & MDD & 27.24 & 252.63 & 46.67 \\
\hline Isopentenylpyrophosphate isomerase & 5.3.3.2 & IPI & 81.66 & 115.06 & 112.65 \\
\hline Geranylgeranyl pyrophosphate synthase & 2.5.1.29 & GGPS & 63.48 & 106.71 & 136.29 \\
\hline Geranylgeranyl diphosphate synthase & 2.5.1.1 & GGR & 178.22 & 1283.19 & 586.18 \\
\hline Farnesyl diphosphate synthase & 2.5.1.10 & FPS & 18.93 & 252.86 & 54.37 \\
\hline Squalene synthase & 2.5.1.21 & SS & 57.08 & 435.07 & 63.94 \\
\hline Squalene epoxidase & 1.14.13.132 & SE & 53.97 & 1690.83 & 118.00 \\
\hline$\beta$-amyrin synthase & 5.4.99.39 & AS & 6.47 & 12.30 & 8.34 \\
\hline Dammarenediol-II synthase & 4.2.1.125 & DS & 162.68 & 1526.21 & 124.10 \\
\hline
\end{tabular}

The values in different organs indicate the reads per kilobase of transcript per million reads mapped (RPKM). 
Table 4 Genes involved in triterpene saponin biosynthesis in P. notoginseng

\begin{tabular}{|c|c|c|c|c|}
\hline Gene name & Abbreviation & Length of mRNA (bp) & Location of CDS & Accession number \\
\hline Acetyl-CoA C-acetyltransferase & AACT & 1816 & $194-1420$ & KJ804173 \\
\hline 3-hydroxy-3-methylglutaryl coenzyme A synthase & HMGS & 1986 & $388-1797$ & KJ804167 \\
\hline 3-hydroxy-3-methylglutaryl coenzyme A reductase & HMGR & 3947 & 198-1967 & KJ804166 \\
\hline Mevalonate kinase & MK & 1767 & $207-1370$ & KJ804176 \\
\hline Phosphomevalonate kinase & PMK & 2078 & $277-1806$ & KJ804170 \\
\hline Mevalonate diphosphate decarboxylase & MDD & 2658 & $386-1648$ & KJ804169 \\
\hline Isopentenyl diphosphate isomerase & IPI & 1246 & 119-1042 & KJ804168 \\
\hline Geranylgeranyl diphosphate synthase & GGR & 1826 & $368-1474$ & KJ804178 \\
\hline Geranylgeranyl pyrophosphate synthase* & GGPS & 1023 & $1-885$ & KJ804179 \\
\hline Farnesyl diphosphate synthase & FPS & 1389 & $141-1169$ & KJ804175 \\
\hline Squalene synthase & SS & 1719 & 284-1531 & KJ804172 \\
\hline Squalene epoxidase & SE & 2054 & $104-1726$ & KJ804171 \\
\hline Beta-amyrin synthase* & AS & 2291 & $1-2130$ & KJ804177 \\
\hline Dammarenediol-II synthase & DS & 2631 & $117-2426$ & KJ804174 \\
\hline
\end{tabular}

*Partial CDS at the $5^{\prime}$ end.

transcriptome, one unigene annotated as CYP716A53v2 was found and subsequent sequence analysis showed that it was the full-length homologue in $P$. notoginseng. Figure 5 shows the alignment of the predicted protein sequences of CYP716A53v2 from $P$. notoginseng, $P$. ginseng and $P$. quinquefolius. The accession numbers of the sequences are JX036031 for P. ginseng and KC190491 for P. quinquefolius in the GenBank database. All three CYP450 genes had 469 amino acid residues and showed $98 \%$ identity between $P$. notoginseng and $P$. ginseng, 96\% identity between $P$. notoginseng and $P$. quinquefolius, and $97 \%$ identity between $P$. ginseng and $P$. quinquefolius. It was also very encouraging to find that the expression level of the putative CYP716A53v2 in the root (RPKM value 414.91) was much higher than that in the leaf (RPKM value 1.83 , $P<0.001$ ) or flower (RPKM value 4.62, $P<0.001$ ) of $P$. notoginseng (Figure 6). To confirm the differential expression of the putative CYP716A53v2 gene, we analysed its expression level in different tissues by real-time PCR. The result obtained was consistent with the RPKM values, showing that the expression of the putative CYP716A53v2 in roots was significantly higher than in leaves or flowers (Figure 6). This may

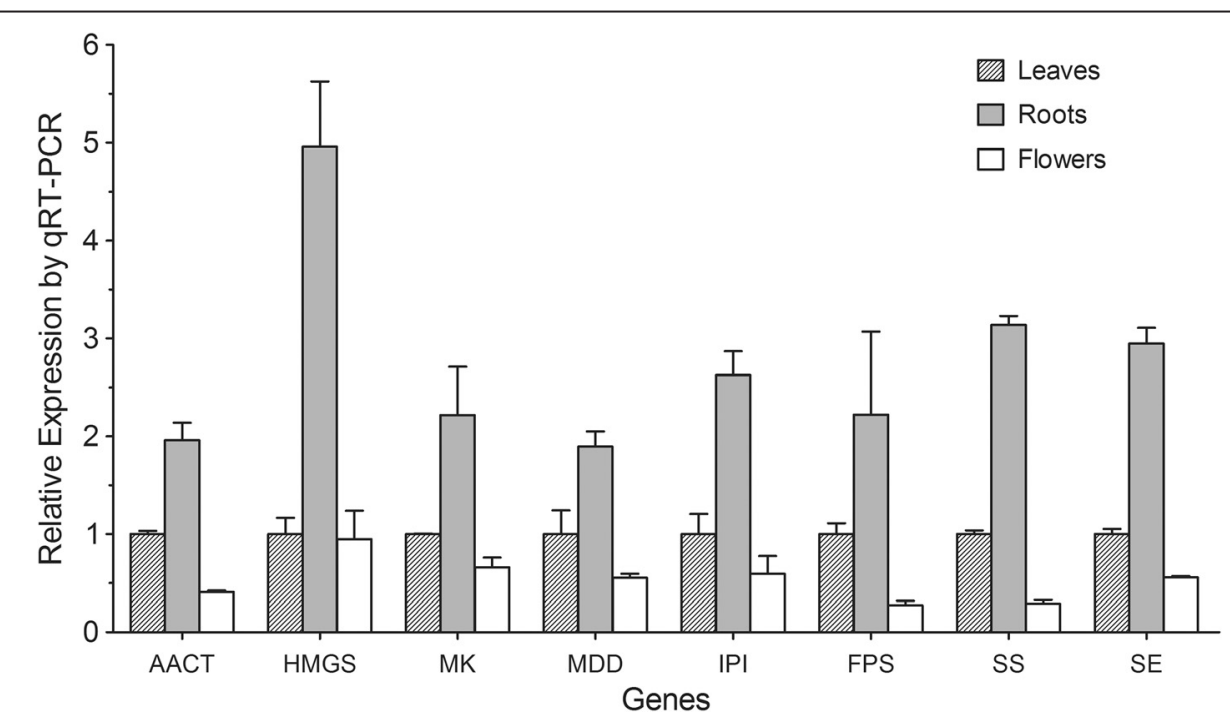

Figure 4 Real-time PCR analysis of selected genes involved in triterpene saponin biosynthesis. Real-time PCR was used to validate the expression levels of selected genes revealed by RNA-seq. Abbreviations: AACT, acetyl-CoA acetyltransferase; HMGS, HMG-CoA synthase; MK, mevalonate kinase; MDD, mevalonate diphosphate decarboxylase; IPI, isopentenylpyrophosphate isomerase; FPS, farnesyl diphosphate synthase; SS, squalene synthase; $\mathrm{SE}$, squalene epoxidase. 


\begin{tabular}{|c|c|c|}
\hline P.notoginseng & MDLFISSQLLLLLVFCLFLFWNFKPSSQNKLPPGKTGWPIIGETLEFISWGQKGNPEKFVTQRMKKYSPD & 70 \\
\hline P.ginseng & MDLFISSQLLLLLVFCLFLFWNFKPSSQNKLPPGKTGWPI IGETLEFISCGQKGNPEKFVTQRMNKYSPD & 70 \\
\hline P.quinquefolius & IPLWNFKPSSQNKLPPGKTGWPI IGETLEFISCGQKGNPEKFVTQRMKKYSPD & 70 \\
\hline P.notoginseng & VETTSLAGEKMVVFCGASGNKFIFSNENKLVVSWWPPAISKILTATI PSVEKSKALRSLIVEFLKPEALH & 140 \\
\hline P.ginseng & VETTSLAGEKMVVFCGASGNKFIFSNENKLVVSWWPPAISKILTATIPSVEKSKALRSLIVEFLKPEALH & 140 \\
\hline P.quinquefolius & VFTTSLAGEKMVVFCGASGNKFIFSNENKLVVSWWPPAISKILTATI PSVEKSKALRSLIVEFLKPEALH & 140 \\
\hline P.notoginseng & KFISVMDRTTRQHFEAKKNGSTEVKAFAMSETLTFELACWLLFS INDPVQVQKLSHLFEKVKAGLLSLPL & 210 \\
\hline P.ginseng & KFISVMDRTTRQHFEDKWNGSTEVKAFAMSESLTFELACWLLFS INDPVQVQKLSHLFEKVKAGLLSLPL & 210 \\
\hline P.quinquefolius & KFISVMDRTTRQHFEDKWNGSTEVKAFAMSESLTFELACWLLFS INDPVQVQKLSHLFEKVKAGLLSLPL & 210 \\
\hline P.notoginseng & NFPGTAFNRGIKAANLIRKELSVVIKQRRSDKSETRKDLLSHVMISNGEGEKFFSEMDIADVVLNILIAS & 280 \\
\hline P.ginseng & NFPGTAFNRGIKAANLIRKELSVVIKQRRSDKIQTRRDLLSHVMLSNGEGEKFFSEMDIADVVLNLLIAS & 280 \\
\hline P.quinquefolius & NFPGTAFNRGIKAANLIRKELSVMIKQRRSDKLQTRKDLLSHVMLSNGEGEKFFSEMDIADVVLNLLIAS & 280 \\
\hline P.notoginseng & HDTTSSAMGSVYFLADHPHIYAKVLTEQMEIAKSKGA GELLSWEDIKRMKYSRNVINEAMRLVPPSQGG & 350 \\
\hline P.ginseng & HDTTSSAMGSVVYFLADHPHIYAKVLTEOMEIAKSKGA EELLSWEDIKRMKYSRNVINEAMRLVPPSQGG & 350 \\
\hline P.quinquefolius & HDTTSSAMGSVVYFLADHPHIYAKVLTEQMEIAKSKGA GELLSWEDIKRMKYSRNVINEAMRLVPPSQGG & 350 \\
\hline P.notoginseng & FKVVTSKF SYANF'I I PKGWKI FWSVYSTHKDPKYFKNPEEFDPSRFEGDGPMPFTFIPFGGGPRMCPGSE & 420 \\
\hline P.ginseng & FKVVTSKFSYANFIIPKGWKIFWSVYSTHKDPKYFKNPEEFDPSRFEGDGPMPFTFIPEGGGPRMCPGSE & 420 \\
\hline P.quinquefolius & FKVVTSKF SYANFI I PKGKK I FWSVYSTHKDPKYFKNPEEFDPSRFEGDGPMPFTFIPFGGGPRMCPGSE & 420 \\
\hline P.notoginseng & FARLEVLIFMHHLVTNFRWEKVFPNEKI IYTPFPFPENGLPIRLSPCTL & 469 \\
\hline P.ginseng & FARLEVLI FMHHLVTNFKWEKVFPNEKIIYTPEPFPENGLPIRLSPCTL & 469 \\
\hline P.quinquefolius & FARLEVLIFMHHLVTNFKWEKVFPNEKI IYTPFPFPENGLPIRLSPCTL & 469 \\
\hline \multicolumn{3}{|c|}{$\begin{array}{l}\text { Figure } 5 \text { Alignment of CYP716A53v2 amino acid sequences from P. notoginseng, P. ginseng and P. quinquefolium. Alignment results } \\
\text { showed 98\% identity between P. notoginseng and P. ginseng, 96\% identity between P. notoginseng and P. quinquefolius, and } 97 \% \text { identity } \\
\text { between P. ginseng and P. quinquefolius. }\end{array}$} \\
\hline
\end{tabular}

explain, at least partially, why the root has a much higher concentration of protopanaxatriol-type saponins. Moreover, this result also indicates that the abundance of target genes in our transcriptomes closely reflects the actual gene expression level.
Identification of candidate genes involved in alkaloid biosynthesis

Alkaloids, which are found in about $20 \%$ of plant species, are a diverse group of low-molecular-weight compounds acting as poisonous agents in the defence of plants against
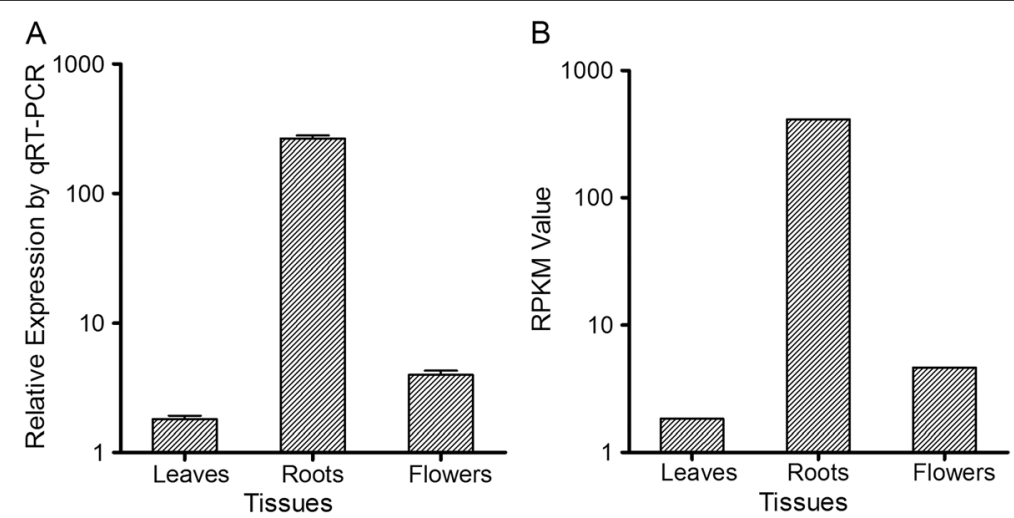

Figure 6 Expression levels of target CYP450s in different $P$. notoginseng tissues. Comparison of relative abundance of target CYP450 unigenes in the leaf, root and flower according to real time PCR (A) and RPKM values (B). 
herbivores and pathogens [19]. P. notoginseng grows suboptimally in direct sunlight and so is often planted under tree canopies, but the shady and humid growing conditions favour infection by numerous phytopathogens, which can cause root rot, black spot or round spot diseases [18].

In this study, three pathways involved in alkaloid biosynthesis were found in our transcriptomes (Table 2), including isoquinoline alkaloid biosynthesis (KEGG pathway entry ko00950), indole alkaloid biosynthesis (ko00901) and tropane, piperidine and pyridine alkaloid biosynthesis (ko00945). The annotation results from the three transcriptomes were used to identify genes encoding enzymes involved in various alkaloid biosynthetic pathways. In total, 72 unigenes were assigned to six enzymes involved in alkaloid biosynthesis (Additional file 7). According to the RPKM values of the genes encoding enzymes involved in alkaloid biosynthesis in leaves, roots and flowers shown in Table 5, most of the enzymes were expressed at the lowest level in roots, in particular polyphenol oxidase (PPO), which has a role in plant resistance to stress and pathogens. It is notable that wounding and herbivore attacks have also been shown to induce PPO activity [30]. PPO activation is thought to involve proteolytic processing, but many mature PPOs appear to remain in a latent form [31]. Root rot disease is the most damaging disease that can plague $P$. notoginseng over its long growth period, leading to production loss and quality reduction. It is mainly caused by fungal pathogens such as Cylindrocarpon destructans, Cylindrocarpon didymum and Fusarium solani [32]. In this study, the expression levels of aspartate transaminase, strictosidine synthase and histidinol-phosphate transaminase in different tissues were validated by real-time PCR (Figure 7). Further investigation of the relationship between alkaloid biosynthesis genes and plant defence should provide novel insights into the complex disease resistance mechanisms of $P$. notoginseng.

\section{Conclusions}

$P$. notoginseng is a widely used medicinal herb. In this study, leaf, root and flower transcriptomes from $P$. notoginseng are presented. The resulting unigene dataset will provide a large number of transcripts for gene discovery and genetic analyses in this medicinal plant.
Notably, many genes involved in triterpene saponin biosynthesis were identified in this study. More importantly, a large number of unigenes were annotated as CYP450s and GTs. In summary, this study provides comprehensive information on the transcriptional regulation of functionally important genes in P. notoginseng.

\section{Methods}

\section{Plant materials}

Growing 3-year-old P. notoginseng was randomly collected from the field of a $P$. notoginseng commercial planting base in Wenshan County, Yunnan Province, China on September 2, 2012. The highest/lowest temperature on that day was $28 / 20^{\circ} \mathrm{C}$, and the relative humidity was $78 \%$. The soil of the field was a sandy loam soil with a $\mathrm{pH}$ value of 5.5-7.0. After cleansing, the leaves, roots and flowers were collected separately, cut into small pieces, immediately frozen in liquid nitrogen, and stored at $-80^{\circ} \mathrm{C}$ until further processing.

\section{RNA extraction}

mRNA isolation, cDNA library construction and sequencing were performed by the Beijing Genomics Institute (BGI) (Shenzhen, China). Briefly, total RNA was extracted from each tissue using TRIzol reagent (Invitrogen, Burlington, ON, Canada) and digested with DNase I (Takara, Dalian, China) according to the manufacturer's protocol. Next, Oligo (dT) magnetic beads were used to isolate mRNA from the total RNA. By mixing with fragmentation buffer, the mRNA was then broken into short fragments.

\section{cDNA synthesis and sequencing}

The cDNA was synthesized using the mRNA fragments as templates. The short fragments were purified and resolved with EB buffer for end repair and single nucleotide A (adenine) addition, and then connected with adapters. Suitable fragments were selected for PCR amplification as templates. During the quality control steps, an Agilent 2100 Bioanalyzer (Agilent Technologies, Redwood City, CA, USA) and ABI StepOnePlus Real-Time PCR System (Life Technologies, Grand Island, NY, USA) were used for quantification and qualification of the sample library. Each

Table 5 Discovery of unigenes involved in alkaloid biosynthesis in $P$. notoginseng

\begin{tabular}{lllll}
\hline Enzymes & EC number & Leaves & Roots & Flowers \\
\hline Aspartate transaminase & 2.6 .1 .1 & 234.19 & 173.03 & 125.11 \\
Primary-amine oxidase & 1.4 .3 .21 & 153.62 & 35.36 & 211.14 \\
Strictosidine synthase & 4.3 .3 .2 & 140.37 & 21.74 & 121.67 \\
Polyneuridine-aldehyde esterase & 3.1 .1 .78 & 21.37 & 20.85 & 34.13 \\
Histidinol-phosphate transaminase & 2.6 .1 .9 & 26.40 & 17.70 & 26.16 \\
Polyphenol oxidase & 1.10 .3 .1 & 143.53 & 145.64 \\
\hline
\end{tabular}

The values in different organs indicate the reads per kilobase of transcript per million reads mapped (RPKM). 


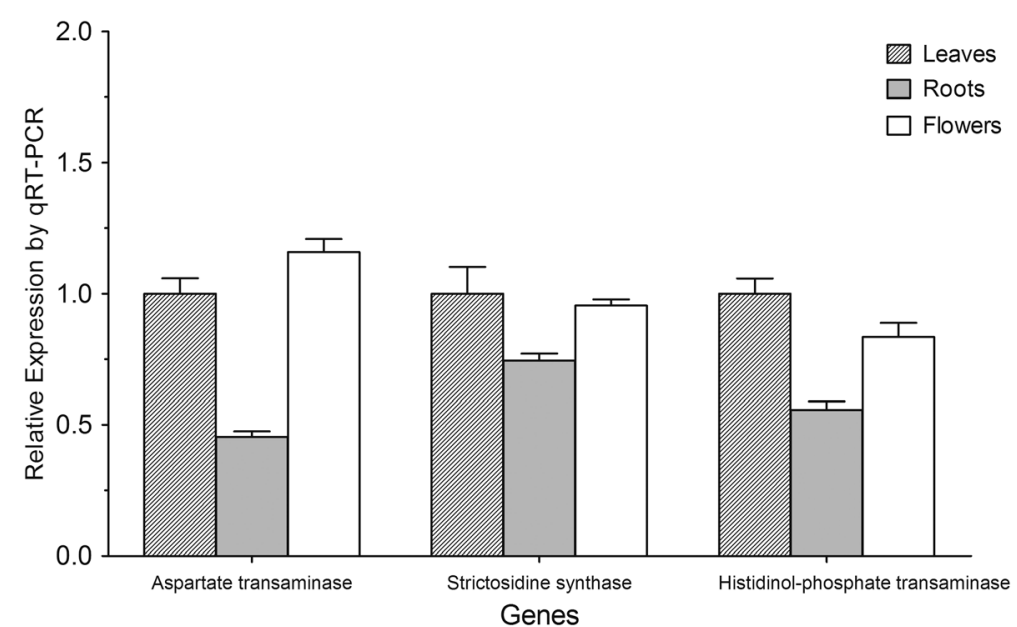

Figure 7 Real-time PCR analysis of genes involved in the alkaloid pathway. The expression levels of selected genes in the alkaloid pathway were validated by real-time PCR.

cDNA library was sequenced in a single lane of the Illumina $\mathrm{HiSeq}^{\mathrm{TM}} 2000$ system using paired end protocols according to the manufacturer's instructions at the Beijing Genomics Institute (BGI) (Shenzhen, China). The amount of reads generated per sample was 5-8 Gb to obtain deep coverage of transcripts for de novo assembly [33]. As determined by SAMtools [34], the average depth of the generated $P$. notoginseng transcriptomes was over $130 \times$.

\section{De novo assembly and sequence annotation}

The raw reads dataset was first processed to remove the reads with adaptors or containing more than five unknown ('N') nucleotides. Next, the low quality reads (defined as reads having more than $20 \%$ of bases with quality $\leq 10$ ) were trimmed. We used the Trinity software (release20121005) [20] for de novo assembly of the high-quality cleaned reads, and then used TGICL [21] followed by the Phrap [22] assembler to remove the redundant Trinitygenerated contigs.

The resulting unigenes were annotated by BLASTx [35] searches against the NCBI nr (ftp://ftp.ncbi.nih.gov/ blast/db/FASTA/nr.gz), UniProt protein (http://www. uniprot.org/downloads), TAIR (http://www.arabidopsis. org/index.jsp), ITAG (http://solgenomics.net/organism/ Solanum_lycopersicum/genome) and PlantCyc (ftp://ftp. plantcyc.org/Pathways/BLAST_sets/) databases with a cut-off value of 1e-5. The top hit was extracted for each unigene.

Groups of orthologous proteins were identified using the OrthoMCL algorithm [24]. To gain an overview of gene networks, KEGG pathway information was assigned to each unigene, based on similarity with the KEGG database [36] using a BLAST search with a cut-off value of $1 \mathrm{e}-5$. The KEGG analysis output included enzyme commission (EC) numbers and KEGG orthology (KO) numbers.

\section{Digital gene expression profiling}

The high-quality reads were aligned to the assembled unigenes with the BWA program [37]. An RPKM value was calculated for each unigene in each tissue of $P$. notoginseng. The RPKMs of all annotated isoforms for the same gene were summed as the RPKM of that gene. Differential expression of unigenes was calculated with a threshold of $P$ value $<0.001$ and 2 -fold change.

\section{Real-time PCR analysis}

RNA was isolated from different $P$. notoginseng tissues and reverse-transcribed to single-strand cDNA using the Super Script ${ }^{\mathrm{TM}}$ III First-Strand Synthesis System (Invitrogen $^{\mathrm{T}}$, USA). Quantitative reactions were performed on the Real-Time PCR Detection System (ABI 7500, Applied Biosystems, USA) using SYBRR Premix Ex Taq ${ }^{\mathrm{Tm}}$ II (Takara Biotechnology, China). The reaction mixture $(20 \mu \mathrm{L})$ contained $2 \times$ SYBRR Premix Ex Taq ${ }^{\mathrm{TM}} \mathrm{II}, 0.4 \mu \mathrm{M}$ each of the forward and reverse primers, and $2 \mu \mathrm{L}$ of template cDNA. PCR amplification was performed under the following conditions: $95^{\circ} \mathrm{C}$ for $30 \mathrm{~s}$, followed by 40 cycles of $95^{\circ} \mathrm{C}$ for $5 \mathrm{~s}$ and $60^{\circ} \mathrm{C}$ for $34 \mathrm{~s}$, and with a dissociation stage of $95^{\circ} \mathrm{C}$ for $15 \mathrm{~s}, 60^{\circ} \mathrm{C}$ for $60 \mathrm{~s}$ and $95^{\circ} \mathrm{C}$ for $15 \mathrm{~s}$. All primers used in this study are listed in Additional file 8 . The relative gene expression was calculated with the ${ }^{{ }^{\Delta}} \mathrm{CT}$ method. For each sample, the mRNA levels of the target genes were normalized to that of the actin mRNA. These experiments were repeated using three biological replications. 


\section{Ethical statement}

There is no conflict of interest. An exemption from requiring ethics approval has been granted from the Joint Chinese University of Hong Kong - New Territories East Cluster Clinical Research Ethics Committee.

\section{Availability of supporting data}

All RNA sequences and raw reads data are available under accession numbers KJ804166 to KJ804179, KF935232 and BioProject accession PRJNA228978.

\section{Additional files}

Additional file 1: Length distribution of contigs and unigenes from leaves, roots and flowers of $P$. notoginseng. PDF document of the length distribution of contigs and unigenes.

Additional file 2: Summary of annotation statistics of the leaves, roots and flowers using various databases. PDF document of the summary of annotation.

Additional file 3: Top 10 most highly expressed transcripts in the $P$. notoginseng root transcriptome. PDF document of the annotation results of the top 10 highly expressed unigenes in the root.

Additional file 4: Number of unigenes assigned to KEGG biochemical pathways in $P$. notoginseng. PDF document of the number of unigenes assigned to KEGG biochemical pathways.

Additional file 5: Summary of unigenes annotated as CYP450. XLSX document of the summary of unigenes annotated as CYP450.

Additional file 6: Summary of unigenes annotated as glycosyltransferase. XLSX document of the summary of unigenes annotated as glycosyltransferase.

Additional file 7: Summary of unigenes encoding enzymes involved in the alkaloid pathway. XLSX document of the annotation results of unigenes encoding enzymes involved in the alkaloid pathway.

Additional file 8: List of real-time PCR primer sequences.

PDF document of the list of real-time PCR primer sequences.

\section{Abbreviations}

AS: $\beta$-amyrin synthase; BLAST: Basic local alignment search tool; bp: Base pair; BWA: Burrows-Wheeler Alignment Tool; cDNA: Complementary DNA; CYP450: Cytochrome P450; DS: Dammarenediol-II synthase; EC: Enzyme commission; GT: Glucosyltransferase; HMGS: Hydroxymethyl glutaryl CoA synthase; ITAG: Tomato genome database; KEGG: Kyoto encyclopaedia of genes and genomes; KO: KEGG orthology; MVA: Mevalonic acid; NCBI: National Centre for Biotechnology Information; Nr: Non-redundant protein database; PPO: Polyphenol oxidase; RPKM: Reads per kilobase of transcript per million reads mapped; SRA: Sequence Read Archive; TAIR: The Arabidopsis Information Resource; TGICL: TGI Clustering Tool.

\section{Competing interests}

The authors declare that they have no competing interests.

\section{Authors' contributions}

SKT, SML and WFC participated in the design, execution and evaluation of the study. BRY, HFZ, GCL and XFL performed the sample collection, RNA extraction, CDNA sample preparation and real-time PCR. MHL, KYY, JSK, SZ, SML and SKT contributed to the data analysis and discussion of the results. MHL drafted the initial manuscript and all members contributed to the preparation of the final manuscript. All authors read and approved the final manuscript.

\section{Acknowledgements}

This project was supported by the Scheme B funding of the project 'Establishment of the Centre for Microbial Genomics and Proteomics' and the Scheme D funding of the project 'Enhancing the capabilities and strengthening the research personnel of CUHK in Bioinformatics' of the
Focused Investment Scheme of The Chinese University of Hong Kong. This project was also supported by the Overseas and Hong Kong, Macau Young Scholars Collaborative Research Fund by the Natural National Science Foundation of China (Grant No. 81328025) and Science and Technology Development Fund of Macau SAR (Ref. No. 014/2011/A1 and 058/2009).

\section{Author details}

${ }^{1}$ School of Biomedical Sciences, The Chinese University of Hong Kong, Hong Kong, China. ${ }^{2}$ Hong Kong Bioinformatics Centre, The Chinese University of Hong Kong, Hong Kong, China. ${ }^{3}$ State Key Laboratory of Quality Research in Chinese Medicine and Institute of Chinese Medical Sciences, Macao, China. ${ }^{4}$ Beijing Genomics Institute - Shenzhen, Shenzhen 518083, China. ${ }^{5}$ The State Key Laboratory of Agrobiotechnolgy, The School of Life Sciences, The Chinese University of Hong Kong, Hong Kong, China. ${ }^{6}$ Present correspondence: Groken Bioscience,

Hong Kong, China.

Received: 25 June 2014 Accepted: 23 March 2015

Published online: 03 April 2015

\section{References}

1. Briskin DP. Medicinal plants and phytomedicines. Linking plant biochemistry and physiology to human health. Plant Physiol. 2000;124(2):507.

2. World Health O. WHO Monographs on Selected Medicinal Plants, vol. 1. 2009.

3. Son HY, Han HS, Jung HW, Park YK. Panax notoginseng attenuates the infarct volume in rat ischemic brain and the inflammatory response of microglia. J Pharmacol Sci. 2009;109(3):368

4. Li H, Deng CQ, Chen BY, Zhang SP, Liang Y, Luo XG. Total saponins of Panax notoginseng modulate the expression of caspases and attenuate apoptosis in rats following focal cerebral ischemia-reperfusion. J Ethnopharmacol. 2009;121(3):412.

5. Yang CY, Wang J, Zhao Y, Shen $L$, Jiang $X$, Xie ZG, et al. Anti-diabetic effects of Panax notoginseng saponins and its major anti-hyperglycemic components. J Ethnopharmacol. 2010;130(2):231

6. Xiang H, Liu Y, Zhang B, Huang J, Li Y, Yang B, et al. The antidepressant effects and mechanism of action of total saponins from the caudexes and leaves of Panax notoginseng in animal models of depression. Phytomedicine. 2011;18(8-9):731.

7. State Administration of Traditional Chinese M. Zhong Hua Ben Cao, vol. 5. Shanghai: Shanghai Science and Technology Press; 1999.

8. Francis G, Levavi-Sivan B, Avitan A, Becker K. Effects of long term feeding of Quillaja saponins on sex ratio, muscle and serum cholesterol and LH levels in Nile tilapia (Oreochromis niloticus (L)). Comp Biochem Physiol C Toxicol Pharmacol. 2002:133(4):593.

9. $\mathrm{Ng}$ TB. Pharmacological activity of sanchi ginseng (Panax notoginseng). J Pharm Pharmacol. 2006:58(8):1007.

10. Park J, Rhee D, Lee Y. Biological activities and chemistry of Saponins from Panax ginseng C. A. Meyer. Phytochem Rev. 2005:4(2-3):159-75.

11. Wan JB, Yang FQ, Li SP, Wang YT, Cui XM. Chemical characteristics for different parts of Panax notoginseng using pressurized liquid extraction and HPLC-ELSD. J Pharm Biomed Anal. 2006:41(5):1596.

12. Fan TP, Yeh JC, Leung KW, Yue PY, Wong RN. Angiogenesis: from plants to blood vessels. Trends PharmacolSci. 2006:27(6):297.

13. Furukawa T, Bai CX, Kaihara A, Ozaki E, Kawano T, Nakaya Y, et al. Ginsenoside Re, a main phytosterol of Panax ginseng, activates cardiac potassium channels via a nongenomic pathway of sex hormones. Mol Pharmacol. 2006:70(6):1916

14. Wang C-Z, McEntee E, Wicks S, Wu J-A, Yuan C-S. Phytochemical and analytical studies of Panax notoginseng (Burk.) F.H. Chen. J Nat Med. 2006;60(2):97-106.

15. Haralampidis K, Trojanowska M, Osbourn AE. Biosynthesis of triterpenoid saponins in plants. Adv Biochem Eng Biotechnol. 2002;75:31

16. Wan JB. Chemical investigation of saponins in different parts of Panax notoginseng by pressurized liquid extraction and liquid chromatography-electrospray ionization-tandem mass spectrometry. Molecules. 2012;17(5):5836.

17. Luo H, Sun C, Sun Y, Wu Q, Li Y, Song J, et al. Analysis of the transcriptome of Panax notoginseng root uncovers putative triterpene saponin-biosynthetic genes and genetic markers. BMC Genomics. 2011:12 Suppl 5:S5.

18. Guo H, Cui X, An N, Cai G. Sanchi ginseng (Panax notoginseng (Burkill) F. H. Chen) in China: distribution, cultivation and variations. Genet Resour Crop Evol. 2010;57(3):453-60. 
19. Facchini PJ. Alkaloid biosynthesis in plants: biochemistry, cell biology, molecular regulation, and metabolic engineering applications. Annu Rev Plant Physiol Plant Mol Biol. 2001;52:29.

20. Grabherr MG, Haas BJ, Yassour M, Levin JZ, Thompson DA, Amit I, et al. Full-length transcriptome assembly from RNA-Seq data without a reference genome. Nat Biotechnol. 2011;29(7):644.

21. Pertea G, Huang X, Liang F, Antonescu V, Sultana R, Karamycheva S, et al. TIGR Gene Indices clustering tools (TGICL): a software system for fast clustering of large EST datasets. Bioinformatics. 2003;19(5):651.

22. de la Bastide M, McCombie WR. Assembling genomic DNA sequences with PHRAP. CurrProtocBioinformatics. 2007;Chapter 11:Unit11.14. doi:10.1002/ 0471250953.bi1 104s17.

23. Li C, Zhu Y, Guo X, Sun C, Luo H, Song J, et al. Transcriptome analysis reveals ginsenosides biosynthetic genes, microRNAs and simple sequence repeats in Panax ginseng C. A. Meyer. BMC Genomics. 2013;14(1):245.

24. Fischer S, Brunk BP, Chen F, Gao X, Harb OS, lodice JB, et al. Using OrthoMCL to assign proteins to OrthoMCL-DB groups or to cluster proteomes into new ortholog groups. CurrProtocBioinformatics. 2011; Chapter 6:Unit 6.12.11.

25. Tansakul P, Shibuya M, Kushiro T, Ebizuka Y. Dammarenediol-II synthase, the first dedicated enzyme for ginsenoside biosynthesis, in Panax ginseng. FEBS Lett. 2006;580(22):5143.

26. Han JY, Kim HJ, Kwon YS, Choi YE. The Cyt P450 enzyme CYP716A47 catalyzes the formation of protopanaxadiol from dammarenediol-II during ginsenoside biosynthesis in Panax ginseng. Plant Cell Physiol. 2011;52(12):2062.

27. Sun C, Li Y, Wu Q, Luo H, Sun Y, Song J, et al. De novo sequencing and analysis of the American ginseng root transcriptome using a GS FLX Titanium platform to discover putative genes involved in ginsenoside biosynthesis. BMC Genomics. 2010;11:262.

28. Yendo AC, de Costa F, Gosmann G, Fett-Neto AG. Production of plant bioactive triterpenoid saponins: elicitation strategies and target genes to improve yields. Mol Biotechnol. 2010;46(1):94.

29. Han JY, Hwang HS, Choi SW, Kim HJ, Choi YE. Cytochrome P450 CYP716A53v2 catalyzes the formation of protopanaxatriol from protopanaxadiol during ginsenoside biosynthesis in Panax ginseng. Plant Cell Physiol. 2012;53(9):1535.

30. Mayer AM. Polyphenol oxidases in plants and fungi: going places? A review. Phytochemistry. 2006;67(21):2318.

31. Virador VM, Reyes Grajeda JP, Blanco-Labra A, Mendiola-Olaya E, Smith GM, Moreno A, et al. Cloning, sequencing, purification, and crystal structure of Grenache (Vitis vinifera) polyphenol oxidase. J Agric Food Chem. 2010;58(2):1189.

32. Ma L, Cao Y, Cheng M, Huang Y, Mo M, Wang Y, et al. Phylogenetic diversity of bacterial endophytes of Panax notoginseng with antagonistic characteristics towards pathogens of root-rot disease complex. Antonie van Leeuwenhoek. 2013;103(2):299-312.

33. Chow KS, Ghazali AK, Hoh CC, Mohd-Zainuddin Z. RNA sequencing read depth requirement for optimal transcriptome coverage in Hevea brasiliensis. BMC Res Notes. 2014;7:69.

34. Li H, Handsaker B, Wysoker A, Fennell T, Ruan J, Homer N, et al. The Sequence Alignment/Map format and SAMtools. Bioinformatics. 2009;25(16):2078,

35. Altschul SF, Gish W, Miller W, Myers EW, Lipman DJ. Basic local alignment search tool. J Mol Biol. 1990;215(3):403.

36. Ogata H, Goto S, Sato K, Fujibuchi W, Bono H, Kanehisa M. KEGG: Kyoto Encyclopedia of genes and genomes. Nucleic Acids Res. 1999;27(1):29.

37. Li H, Durbin R. Fast and accurate short read alignment with BurrowsWheeler transform. Bioinformatics. 2009;25(14):1754.

\section{Submit your next manuscript to BioMed Central and take full advantage of:}

- Convenient online submission

- Thorough peer review

- No space constraints or color figure charges

- Immediate publication on acceptance

- Inclusion in PubMed, CAS, Scopus and Google Scholar

- Research which is freely available for redistribution 